\title{
Atitudes dos agentes comunitários de saúde relacionadas à saúde mental
}

\author{
Attitudes of community health agents related to mental health
}

Actitudes de los agentes de salud comunitarios relacionados con la salud mental

José Luis da Cunha Pena ${ }^{1}$, Veronica Batista Cambraia Favacho ${ }^{1 *}$, Maria Silvia da Costa Silva ${ }^{2}$, Cassio Diogo Almeida Monteiro ${ }^{3}$, Emília Nazaré Menezes Ribeiro Pimentel ${ }^{4}$, Raissa dos Santos Flexa ${ }^{1}$, Carla Emanuela Xavier Silva ${ }^{1}$, Ailson Soares de Almeida1', Gabriella Furtado Monteiro ${ }^{1}$, Márcia Aparecida Ferreira de Oliveira ${ }^{5,6}$.

\section{RESUMO}

Objetivo: Identificar as atitudes relacionadas à saúde mental em agentes comunitários de saúde pré-pós intervenção de atualização educativa. Métodos: Estudo quase-experimental, do tipo pré-pós com abordagem quantitativa, com uso da Escala de Atitudes Relacionadas à Saúde Mental. Resultados: Após intervenção de atualização educativa, dos nove itens da escala, considerados com atitudes negativas em relação à saúde mental (itens 2, 4, 5, 7, 8, 10, 11, 12 e 13), cinco itens apresentaram redução em sua concordância pelos participantes (56\%). Por outro lado, dos seis itens da escala, considerados com atitudes positivas da escala em relação à saúde mental (itens 1, 3, 6, 9, 14 e 15), cinco itens mostraram aumento em sua concordância pelos participantes (83\%), ou seja, dos quinze itens totais da escala, dez itens obtiveram mudança de atitudes em saúde mental (67\%). Conclusão: Evidenciou-se melhora no conhecimento dos agentes comunitários de saúde após intervenção de atualização educativa sobre as atitudes relacionadas à saúde mental.

Palavras-chave: Atenção primária à saúde, Agentes comunitários de saúde, Saúde mental.

\begin{abstract}
Objective: To identify attitudes related to mental health in community health agents before/after an educational update intervention. Methods: Quasi-experimental study, pre-post type with a quantitative approach, using the Scale of Attitudes Related to Mental Health. Results: After an educational update intervention, of the nine items on the scale, considered to have negative attitudes towards mental health (items 2, 4, 5, 7, 8, 10, 11, 12 and 13), five items showed a reduction in their agreement by the participants (56\%). On the other hand, of the six items of the scale, considered with positive attitudes of the scale towards mental health (items 1, 3, 6, 9, 14 and 15), five items showed an increase in their agreement by the participants (83\%), in other words, of the fifteen total items of the scale, ten items achieved a change in mental health attitudes (67\%). Conclusion: There was an improvement in the knowledge of community health agents after an educational update intervention on attitudes related to mental health.
\end{abstract}

Keywords: Primary health care, Community health agents, Mental health.

\footnotetext{
1 Universidade Federal do Amapá (UNIFAP), Macapá - AP. *E-mail: vc.cambraiafa@unifap.br

2 Secretaria de Saúde do Estado do Amapá (SESA), Macapá - AP.

${ }^{3}$ Faculdade Estácio de Macapá (SEAMA), Macapá - AP.

${ }^{4}$ Conselho Regional de Enfermagem do Amapá (COREN-AP), Macapá - AP.

5 Universidade de São Paulo (USP), São Paulo - SP.

6 Universidade Federal de Santa Maria (UFSM), Santa Maria - RS.
} 


\section{RESUMEN}

Objetivo: Identificar las actitudes relacionadas con la salud mental en agentes comunitarios de salud antes y después de una intervención de actualización educativa. Métodos: Estudio cuasi experimental, tipo antes y después con enfoque cuantitativo, utilizando la Escala de Actitudes Relacionadas con la Salud Mental. Resultados: Tras una intervención de actualización educativa, de los nueve ítems de la escala, considerados con actitudes negativas en relación a la salud mental (ítems 2, 4, 5, 7, 8, 10, 11, 12 y 13), cinco ítems presentaron una reducción de acuerdo con los participantes (56\%). Por otro lado, de los seis ítems de la escala, considerados con actitudes positivas hacia la salud mental (ítems 1, 3, 6, 9, 14 y 15), cinco ítems mostraron un aumento por parte de los participantes (83\%). Por lo tanto, del total de quince ítems de la escala, diez ítems revelaron cambios de actitudes en salud mental (67\%). Conclusión: Hubo una mejora en el conocimiento de los agentes comunitarios de salud luego de una intervención de actualización educativa sobre actitudes relacionadas con la salud mental.

Palabras clave: Atención primaria de salud, Agentes comunitarios de salud, Salud mental.

\section{INTRODUÇÃO}

A saúde mental traz a importância da sua relação com as práticas de cuidados à saúde como tema investigado mundialmente, sobretudo porque a saúde mental está envolta no cuidado da integralidade da pessoa. Entretanto, muito ainda é necessário para avançar no cuidado da pessoa em sofrimento psíquico. De tal modo, pesquisas evidenciam a dificuldade encontrada por profissionais no que se refere à compreensão do processo de cuidado e de atenção psicossocial. Deve-se compreender por que a partir da Reforma Psiquiátrica surge nova perspectiva e prática no cuidar, sendo imperativo a inclusão desse outro olhar na formação profissional e em capacitações nos dispositivos de saúde onde o cuidado em saúde mental acontece (PEIXOTO MM, et al., 2016; VASCONCELOS MGD, et al., 2016).

Neste sentido, o caminho histórico no campo das Políticas Públicas pensadas às pessoas com necessidades de cuidados em saúde mental revela-se importante. Isto se dá quando a Política Nacional de Saúde Mental reconhecidamente tem seu espaço afirmado a partir da Luta Antimanicomial e com a Reforma Psiquiátrica. Logo, o surgimento da Lei 10.216 de abril de 2001, que trata sobre os direitos e a proteção das pessoas em sofrimento mental e reorganiza a proposta assistencial em Saúde Mental e a atenção psicossocial acompanham este processo de transformação com mudanças significativas (TRAJANO MP, et al., 2018).

Assim, a criação dos Centros de Atenção Psicossocial (CAPS) se dá para oferecer cuidado de forma integral às pessoas em sofrimento psíquico. Nesta perspectiva advém a Rede de Atenção Psicossocial (RAPS), de acordo com a Portaria N 3.088, de dezembro de 2011, visando um cuidado em Saúde Mental descentralizado e voltado para o território e para a comunidade. Sob esta égide, nos dias atuais, a atenção básica em saúde vem como dispositivo dentro da RAPS, além da atenção psicossocial estratégica, atenção residencial de caráter transitório, estratégias de desinstitucionalização, atenção de urgência e emergência e atenção hospitalar (TRAJANO MP, et al., 2018).

A RAPS foi criada definindo uma proposta de cuidado à saúde mental onde protagonizam a atenção comunitária, a participação social e não menos importante, a atenção ao usuário de álcool e outras drogas, com uma perspectiva de gestão regional. Diante disso, tais diretrizes visam facilitar o acesso e aumentar 0 número de usuários com a disponibilização de diversas portas de entrada (TRAPÉ TL e CAMPOS RO, 2017).

Desta forma, Barbosa VFB, et al. (2016) refere uma lacuna ao considerar a conjuntura atual do campo da saúde mental brasileira, no que se refere a necessidade mais apoio financeiro e mais espaços de discussão que envolva o pensar crítico no sentido de nova práxi que priorize o cuidado em saúde mental no dia-a-dia dos serviços, potencializando o tecer da rede de atenção psicossocial. Para o autor, atender esta demanda exige um comportamento que não mais naturalize as práticas inversamente desenvolvidas, hospitalocêntricas, medicalizadoras, biologicistas, onde a clínica tradicional obedece o termo originário do adjetivo grego klinikós. 
Partindo destas reflexões, a APS juntamente com todos os outros dispositivos de saúde mental ocupa um lugar relevante para a clínica na rede de cuidados ampliados e inclusivos, considerando que os mesmos devem ter um caminho também pela dimensão clínica, exercendo seu papel terapêutico (DAUMOUS I e ERLICH H, 2017).

Assim, outra contribuição da Atenção Básica para a Política Nacional de Saúde Mental, é que esta, constitui-se como conector entre o acesso e o cuidado dos usuários inseridos no contexto da clínica psicossocial e do adoecimento mental (SOUZA AC, 2015). O acréscimo de A realização de práticas que impliquem em atitudes positivas como as ações de prevenção gera maior impacto na disponibilidade dos profissionais e gestores para efetivar intervenções clínicas com o objetivo de promover saúde por meio das medidas preventivas (COPPOOLSE HL, et al., 2020).

Seguindo esta discussão, a APS como dispositivo da RAPS tem como ferramenta importante a Estratégia Saúde da Família (ESF). E é a partir deste conceito que Camatta MW, et al. (2016) ressalta A ESF espaço primordial para promover cuidados em saúde mental, principalmente, por ser presença efetiva e transformadora em saúde no território e ter a família como seu foco de cuidado.

O cuidado em saúde mental se dá através da aproximação nos diversos níveis de cuidado em saúde. Para tanto, é na dimensão do território que se concretiza, por meio do papel fundamental da Unidade Básica de Saúde e ESF. Esses profissionais integram o serviço de saúde e seus usuários. O agente comunitário de saúde (ACS) tem papel basilar ao atuar nas comunidades, interligando os serviços de saúde à população $e$ assim se reconhecem no significado que a profissão remete, o elo entre o serviço de saúde e a comunidade, o território, promovendo compreensões e vivências ampliadas do conceito de saúde (PINHEIRO LS, et al., 2019).

O convívio no território necessita de compartilhamento de vida no cotidiano; é preciso deixar-se sentir pela vida do outro. Cabe ressaltar, a intermediação desempenhada pelos ACS entre os saberes técnicos e populares e entre a equipe de saúde e a comunidade. Isso ressalta o fato de pertencimento simultaneamente da equipe de saúde e da comunidade; sua práxis é para comunidade e da comunidade; onde os saberes técnico-científicos os alimentam e a cultura local de saúde também os embebem, ao mesmo tempo (SOUZA AC, 2015).

Os ACS na atenção básica representam um importante papel social enquanto atores na capacidade de motivar as pessoas a agir para alcançar determinado objetivo em saúde na comunidade. No entanto, no campo da saúde mental, pode-se considerar que é evidente uma necessidade de educação continuada e incentivo financeiro, pois, a falta de conhecimento introdutório para o exercício profissional limita o processo de trabalho. Para tanto, seria necessário discutir a formação inicial do ACS, compreendendo como estratégia motivadora de mudança nas relações, nos processos, no cuidado em saúde mental e, principalmente, como transformação na oferta dos serviços e na condução do sistema de saúde (PINHEIRO LS, et al., 2019).

Os estudos realizados por Silva-Junior R, et al. (2016) mostraram a qualificação dos ACS em saúde mental como uma lacuna diretamente relacionada a inexistência dessa ação e provavelmente, refletindo em percepções equivocadas, consequentemente sendo um complicador para a prestação do cuidado.

A carência de conhecimento dos profissionais ACS e de enfermagem sobre saúde mental, mais precisamente, comportamento suicida, tem relação direta na falta de preparo para a atuação à pessoas com essas necessidades de cuidados, como intervenções, orientações e encaminhamentos aos serviços especializados de modo assertivo, da mesma maneira na falta de efetividade do compartilhamento do cuidado entre a APS e os demais pontos da RAPS (SILVA PF, et al., 2018).

Em estudo realizado no Amapá, Brasil, com ACS que atuam na atenção básica, os resultados mostraram que o vínculo com o território tem relevância significativa por está apoiado na estratégia de escuta, funcionando como um facilitador na construção de cuidados relacionados às necessidades específicas dos usuários e suas famílias. No entanto, o modelo biomédico pode ser inerente à sua prática, repetindo um comportamento de assistência voltado para a clínica tradicional, tratamento e medicalização da doença, corroborando a ausência de promoção de saúde, contrariando a essência da ESF, especialmente, em saúde mental (SILVA BM, et al., 2020). 
A qualificação dos profissionais da APS pode ser categorizada como mais um obstáculo na prestação do cuidado na atenção domiciliar humanizada e integral, se tornando imperativo os mais diversos tipos de investimentos na formação desses profissionais, como agregar em várias áreas do saber o conhecimento técnico científico (ANDRADE AM, et al., 2017; CASTRO EAB, et al., 2018; PIRES MRGM, et al., 2013; QUEIROZ HAH, et al., 2013; SAVASSI LCM, 2016).

A aproximação da APS, por meio das equipes da ESF, com os demais dispositivos de cuidados à saúde mental, configura uma inovação na organização do trabalho e no acesso à rede de atenção psicossocial, somando-se como mais um recurso para as pessoas que sofrem com transtornos mentais. De tal modo, que a corresponsabilidade seja estabelecida entre os serviços de saúde mental e os da atenção primária à saúde (DAUMOUS I e ERLICH H, 2017).

Diante do contexto, o presente estudo teve como objetivo identificar as atitudes relacionadas à saúde mental em agentes comunitários de saúde pré/pós intervenção de atualização educativa.

\section{MÉTODOS}

Estudo quase-experimental, do tipo pré/pós com abordagem quantitativa. A variável independente (intervenção de atualização teórico educativa) foi manipulada sem randomização ou especificação de grupo controle dos participantes. Nestes estudos do tipo pré/pós, as mensurações são realizadas pré e pós à intervenção feita com os participantes (POLIT DF e BECK CT, 2011). Desse modo, cada profissional assumiu o papel de seu próprio controle para a aferição dos efeitos da intervenção educativa.

Tratando-se de uma pesquisa que envolve informações de pessoas, respeitou-se, no seu desenvolvimento, a Resolução №466/2012, do Conselho Nacional de Saúde que faz orientações e recomendações para realização de estudos com seres humanos, CAAE: 33180620.1.0000.0003 e parecer 4.172.303 do Comitê de Ética em Pesquisa (UNIFAP).

O estudo ocorreu nas dependências de uma instituição de ensino superior, em Macapá, e envolveu os profissionais ACS cadastrados e ativos no trabalho da Estratégia Saúde da Família (ESF), no período de dezembro de 2019. O recrutamento ocorreu pela própria Secretaria de Saúde do Município de Macapá, por meio de convites enviados para todas as equipes de ESF, condicionados à resposta de confirmação de participação. Não foi estabelecido o número de participantes, pois a amostra dependia da disponibilidade dos ACS no período proposto.

O estudo foi conduzido passando por três etapas, com intervalo de minutos a horas entre cada uma delas, a saber: 1) pré intervenção presencial, antes de começar a atualização educativa propriamente dita; 2) logo em seguida-presencial, aplicação da intervenção- presencial, por meio da atualização educativa; 3) pós intervenção-presencial, ao final da atualização educativa para avaliação.

A coleta de dados ocorreu em dois momentos distintos, pré e pós intervenção de atualização educativa. Para isso foi utilizada a Escala de Atitudes em Saúde Mental (EASM), autoaplicável, preenchida pelos participantes (SOUSA PF, et al., 2017).

A EASM é um instrumento no formato Likert, para sua análise, foram atribuídas notas variando de 1 (Discorda plenamente) a 7 (Concorda plenamente). Assim, quanto mais próximo a "1", maior a discordância e quanto mais próximo de "7", maior a concordância com o item da escala.

Os dados foram analisados no software IBM SPSS para Windows, versão 26.0. Na análise das respostas da escala, utilizou-se um escore médio das respostas dos entrevistados em ambos os momentos (pré e pósintervenção); além disso, foram atribuídas as categorias: atitudes negativas em relação à saúde mental (itens $2,4,5,7,8,10,11,12$ e 13) e atitudes positivas da escala em relação à saúde mental (1, 3, 6, 9, 14 e 15). Calculou-se o nível de melhora de atitudes em relação à saúde em cada categoria, em percentual (\%), pela soma dos itens com melhor escores, dividido pelo total de itens de cada categoria, x 100. A categoria geral da escala foi calculada pela média aritmética de todos os escores dos quinze itens da escala. Assim, o nível de mudança de atitudes em saúde mental nas categorias poderia variar de 0 (menor nível de mudança) a $100 \%$ (maior nível de mudança). 
Para comparar os valores dos escores utilizou-se o teste $t$ de Student e para a homogeneidade de variâncias, o teste de Levene, o alfa de Cronbach para consistência interna.

\section{RESULTADOS}

Participaram do estudo no pré-teste 285 ACS, dos quais 112 retornaram para o pós-teste. Em relação aos resultados da EASM a Tabela 1 apresenta o efeito da intervenção por meio da comparação do escore médio dos itens da categoria atitudes negativas em relação à saúde mental (itens 2, 4, 5, 7, 8, 10, 11, 12 e 13). Dos nove itens analisados, em cinco itens houve redução do escore médio; ou seja, houve menor concordância na resposta do item de categoria atitudes negativas em relação à saúde mental (itens $2,7,10,11$ e 13) em $56 \%$ dos escores. Dentre esses, o item "2 - Num tratamento de saúde mental, cabe apenas ao médico indicar o momento da alta" obteve diferença estatisticamente significativa $(p=0,03)$.

Tabela 1 - Análise do efeito da intervenção de atualização educativa da categoria atitudes negativas em relação à saúde mental dos ACS.

\section{Escala de Atitudes em \\ Saúde Mental - EASM}

\begin{tabular}{|c|c|c|c|}
\hline \multicolumn{2}{|c|}{ Escore Médio } & \multirow{2}{*}{$\begin{array}{l}\text { Diferença } \\
\text { Média }\end{array}$} & \multirow[b]{2}{*}{ P-valor ${ }^{*}$} \\
\hline $\begin{array}{c}\text { Pré } \\
(\mathrm{n}=285)\end{array}$ & $\begin{array}{c}\text { Pós } \\
(n=112)\end{array}$ & & \\
\hline
\end{tabular}

\begin{tabular}{|c|c|c|c|c|}
\hline $\begin{array}{l}\text { 2- Num tratamento de saúde mental, cabe apenas ao } \\
\text { médico indicar o momento da alta. }\end{array}$ & 4,78 & 4,12 & $-0,66$ & 0,03 \\
\hline $\begin{array}{l}\text { 4- O doente mental deve ser isolado quando fica } \\
\text { agressivo fisicamente. }\end{array}$ & 3,21 & 3,31 & 0,10 & 0,72 \\
\hline $\begin{array}{l}\text { 5- Só com o consentimento do paciente pode-se } \\
\text { iniciar um tratamento psiquiátrico. }\end{array}$ & 2,60 & 2,87 & 0,27 & 0,29 \\
\hline $\begin{array}{l}\text { 7- O trabalhador dos serviços de saúde mental não } \\
\text { deve se envolver nas lutas por maiores direitos sociais } \\
\text { do doente mental. }\end{array}$ & 3,09 & 2,98 & $-0,11$ & 0,69 \\
\hline $\begin{array}{l}\text { 8- É muito perigoso conviver com um doente mental } \\
\text { em casa. }\end{array}$ & 3,78 & 4,09 & 0,31 & 0,25 \\
\hline $\begin{array}{l}\text { 10- Só um médico psiquiatra é plenamente capaz de } \\
\text { diagnosticar, tratar e compreender a doença mental. }\end{array}$ & 2,99 & 2,82 & $-0,17$ & 0,51 \\
\hline 11- O paciente precisa estar em casa para se tratar. & 2,84 & 2,53 & $-0,31$ & 0,20 \\
\hline $\begin{array}{l}\text { 12- Apenas a psiquiatria é a ciência responsável por } \\
\text { tratar a doença mental. }\end{array}$ & 2,50 & 2,64 & 0,14 & 0,54 \\
\hline $\begin{array}{l}\text { 13- Só se consegue curar a depressão via tratamento } \\
\text { médico. }\end{array}$ & 2,40 & 2,10 & $-0,30$ & 0,15 \\
\hline
\end{tabular}

Legenda: *Teste t de Student.

Fonte: Pena JL, et al., 2021.

Por outro lado, a Tabela 2 mostra os efeitos da intervenção de atualização educativa da categoria atitudes positivas em relação à saúde mental (itens 1, 3, 6, 9, 14 e 15), seis itens avaliados no total, destes, cinco itens apresentaram aumento do escore médio, ou seja, nesses itens, houve maior concordância dos participantes em relação a atitudes positivas à saúde mental (itens 3, 6, 9, 14 e 15) 83\% dos escores. Dentre estes, o item "14- O paciente pode decidir o momento da alta hospitalar" resultou em diferença estatisticamente significativa $(p=0,01)$, com alfa de Cronbach $=0,97$, consistência interna considerada elevada. Desse modo, dos quinze itens totais da escala dez itens obtiveram mudança de atitudes em saúde mental (67\%). 
Tabela 2 - Análise do efeito da intervenção de atualização educativa da categoria atitudes positivas em relação à saúde mental dos ACS.

\begin{tabular}{|c|c|c|c|c|}
\hline \multirow{2}{*}{$\begin{array}{l}\text { Escala de Atitudes em } \\
\text { Saúde Mental - EASM }\end{array}$} & \multicolumn{2}{|c|}{ Escore Médio } & \multirow{2}{*}{$\begin{array}{l}\text { Diferença } \\
\text { Média }\end{array}$} & \multirow{2}{*}{ P-valor } \\
\hline & $\begin{array}{c}\text { Pré } \\
(n=285)\end{array}$ & $\begin{array}{c}\text { Pós } \\
(n=112)\end{array}$ & & \\
\hline $\begin{array}{l}\text { 1- Participar de associações e/ou clubes em prol da } \\
\text { cidadania é direito do doente mental. }\end{array}$ & 6,17 & 5,97 & $-0,20$ & 0,34 \\
\hline $\begin{array}{l}\text { 3- Estudo, trabalho e convívio social são essenciais } \\
\text { para tratar a esquizofrenia. }\end{array}$ & 5,72 & 5,88 & 0,16 & 0,45 \\
\hline $\begin{array}{l}\text { 6- Os vínculos sociais podem tratar um paciente } \\
\text { psiquiátrico. }\end{array}$ & 5,72 & 5,78 & 0,06 & 0,78 \\
\hline 9- O doente mental deve ser tratado na comunidade. & 5,20 & 5,50 & 0,30 & 0,23 \\
\hline $\begin{array}{l}\text { 14- O paciente pode decidir o momento da alta } \\
\text { hospitalar. }\end{array}$ & 2,13 & 2,74 & 0,61 & 0,01 \\
\hline $\begin{array}{l}\text { 15- A doença mental, assim como o seu tratamento, } \\
\text { envolve mais problemas sociais do que orgânicos. }\end{array}$ & 4,95 & 5,41 & 0,46 & 0,06 \\
\hline
\end{tabular}

Legenda: *Teste t de Student.

Fonte: Pena JL, et al., 2021.

\section{DISCUSSÃO}

A Reforma Psiquiátrica afirma o cuidado em Saúde Mental como prática orientada pela valoração da autonomia e dos laços familiares e pela (re)inserção social do ser humano como usuário do serviço de saúde. Identificar as atitudes relacionadas à saúde mental, entre os ACS, no curso de atualização nessa temática, principalmente no contexto da RAPS, deve favorecer a (re)vivência das pessoas com problemas de saúde mental em seus territórios de relações, espaços e possibilidades. A Atenção Primária à Saúde é considerada o principal mecanismo de reorganização do Sistema Único de saúde, preferencialmente por meio da equipe de Estratégia de Saúde da Família, à qual enfrenta o maior desafio de transpor com a concepção tradicionalista de fazer saúde, com a premissa do cuidado e assistência baseados na família e seu contexto social (FARIAS DN, et al., 2018).

Comparando os dois momentos de avaliação dos participantes, estes reduziram sua concordância no item "2", referente à "alta somente pelo profissional médico"; ou seja, os ACS, após a capacitação, concordaram menos com essa afirmativa.

$\mathrm{Na}$ Inglaterra, um estudo com pacientes internados em serviço de saúde mental mostrou que a comparação com a liberação tardia da alta (altas atrasadas em três meses) dessas pessoas se assemelhava à de um grupo de hospitalizações longas. De tal modo, que se faz imperativo debater e propor reflexões sobre essa questão da alta, com o objetivo que ela seja efetivada com sucesso, em um contexto mais amplo de atitudes em saúde mental (GIROLAMO G, et al., 2014; POOLE R, et al., 2014).

Numa perspectiva de fator dificultador no processo de alta, evidencia-se a ausência de apoio e articulação entre a RAPS. Em proposição de reflexão, o processo de alta deve ser visto em uma concepção na qual os profissionais da rede de saúde a efetive com eficácia e em consonância com as políticas públicas pertinentes às demandas de necessidades e integralidade nas práticas de saúde para as pessoas com sofrimento mental (SILVA LL, et al., 2019).

Percebe-se que o que tem dificultado a alta é a falta de apoio e articulação entre a oferta de tratamento contínuo nos hospitais e a própria internação, contribuindo para longa permanência das pessoas nestes serviços (TANIOKA T, et al., 2013). 
Segundo Tanioka T, et al. (2013), Carlotto MS, et al. (2015) e Mcminn BG, et al. (2017), um fator que contribui consideravelmente para um longo tempo de permanência nas instituições de saúde mental são as altas escassas e muitas vezes atrasadas.

Contudo, na contramão dessas evidências, um estudo no sul do Brasil teve, como resultados, grande número de altas e tempo relativamente baixo de permanência no serviço. Este dado apoia a problematização da alta, como está sendo arrazoada e, consequentemente, efetivada por pontos da RAPS, ponderando a transitoriedade e mirando na (re)inserção territorial (TANIOKA T, et al., 2013).

Corroborando o estudo em epígrafe, na perspectiva da alta hospitalar, os ACS, após a atualização em saúde mental, reduziram sua concordância com o item no qual apenas o médico indica o momento da alta em saúde mental. Nesse sentido, Zanardo GL, et al. (2018) também acreditam que o processo de alta deve ter planejamento e articulação entre a RAPS, sobretudo com o usuário, respeitando os anseios e a autonomia deste. Portanto, a efetivação e a continuidade do cuidado e a construção da RAPS mais sólida e resolutiva dependem do vínculo, da parceria e do apoio mútuo entre a equipe de saúde, em seus diversos pontos, inclusive na APS, por meio também do ACS.

O presente estudo converge com o de Mendes VC, et al. (2019), contribuindo para que os profissionais de saúde vislumbrem suas ações diante da alta hospitalar numa reorganização do processo de trabalho e no aprimoramento dos cuidados à saúde, notadamente em saúde mental.

Além disso, a RAPS, trazendo como ponto a APS, torna factível a compreensão da importância e da necessidade de implementar estratégias no cuidado territorializado de saúde mental, centrado no usuário, na família e na rede apoio, não somente na doença, e possibilitando atividades que desenvolvam a prevenção e promoção em saúde mental, efetivando, para o usuário, a continuidade do cuidado na realidade onde ele vive (GONZALES AM, 2015).

Segundo Âdnanes M, et al. (2020), o paciente enfatiza a importância do planejamento de alta e da psicoeducação durante e após a internação, bem como o benefício de planos estruturados, estratégias de enfrentamento, técnicas de automonitoramento e contato próximo com os serviços comunitários locais, além do apoio de colegas e familiares.

De maneira geral, pode-se observar que a estratégia proposta pela atualização em saúde mental acrescenta reflexões que justificam as transformações singulares de atitudes dos participantes do estudo, em relação aos conceitos de: aceitação, autonomia, cuidado no território, direitos humanos, cidadania e atenção psicossocial. Como implicação desse resultado, é necessário reforçar a possibilidade de educação permanente para a equipe da saúde, que fortalece a autonomia e a igualdade entre os trabalhadores (HERMIDA PMV, et al., 2015; XAVIER LN, et al., 2014).

Nesse movimento, a atuação nos mais diversos espaços e campos do Sistema Único de Saúde e nas instituições educacionais se faz desafiadora em formar profissionais da saúde técnicos, críticos e reflexivos, comprometidos com um olhar de cuidado mais humanizado, particularizando as necessidades dos usuários, famílias e comunidades, para assim, compreender os determinantes sociais de saúde que influenciam na qualidade de vida do coletivo e, só então intervir (HERMIDA PMV, et al., 2015; XAVIER LN, et al., 2014).

Nesse sentido, o presente estudo evidencia o papel relevante dos cursos de atualização, por propiciar a construção de espaços que promovam diálogos e reflexões acerca da saúde mental, aos profissionais ACS, como o cuidado em saúde na atenção primária. Assim, de acordo com os avanços para o olhar clínico e nãobiologicista na área da saúde mental, é mister a preocupação com a formação de profissionais que atuam na Atenção Primária à Saúde para que suas atividades profissionais coadunem com as políticas públicas contemporâneas nessa área (MORERA JAC, et al., 2015).

A qualificação dos profissionais constitui-se em uma ação estratégica para a transformação das práticas de saúde. O funcionamento da equipe de saúde da família é diversificado e se caracteriza por ter infinitas variáveis com infinitas conexões entre elas, requerendo qualificação para tanto. Portanto, o diálogo entre as equipes da APS e o conhecimento sobre a Atenção Psicossocial deve ser o foco para um atendimento em 
rede na saúde mental. Todavia, essa práxis ainda é incipiente, com muitas limitações na assistência à essas pessoas com necessidades em saúde mental, uma vez que estes profissionais não são qualificados a contento, evidenciando em suas rotinas de serviço pouco ou nenhum domínio sobre suas funções no cuidado à saúde mental na Atenção Primária à Saúde (CARVALHO MFAA, et al., 2018).

A educação continuada dos profissionais da saúde da APS se apresenta na agregação de conhecimentos e práticas de qualidade, com caráter interdisciplinar e integral para pessoas que têm necessidades dentro do amplo espectro da saúde mental. Desenhada com configurações estratégicas para a reforma do cenário experienciado no SUS e na rede intersetorial, abarca ações inovadoras que incorporam elementos teóricos alinhados com as práticas cotidianas em face dos desafios dentro das prioridades nos cuidados em saúde mental (JORGE MSB, et al., 2017; MITRE SM, et al., 2014).

\section{CONCLUSÃO}

O estudo apresentou limitações como o tamanho amostral diferente para os dois momentos de intervenção e população específica à área de formação em saúde. Evidenciou-se melhora no conhecimento dos agentes comunitários de saúde após intervenção de atualização educativa sobre as atitudes relacionadas à saúde mental. Portanto, as atualizações do conhecimento enriquecem as condutas e práticas dos profissionais, desenvolvendo habilidades na promoção de ações em saúde mental eficazes. Este resultado embasa as ações de intervenções educativas como política institucional para a saúde mental. Assim, espera-se que estes achados subsidiem e instiguem novos estudos, voltados para a mesma temática, uma vez que existe escassez de evidências científicas em relação aos profissionais de saúde e à saúde mental.

\section{REFERÊNCIAS}

1. ÅDNANES M, et al. Planejamento de alta, autogerenciamento e apoio comunitário: estratégias para evitar a rehospitalização psiquiátrica da perspectiva do usuário do serviço. Educação e Aconselhamento do Paciente, 2020; 103(5): 1033 - 40.

2. ANDRADE AM, et al. Atuação do enfermeiro na atenção domiciliar: uma revisão integrativa da literatura. Revista Brasileira de Enfermagem, 2017; 70(1): 210-19.

3. BARBOSA VFB, et al. O cuidado em saúde mental no Brasil: uma leitura a partir dos dispositivos de biopoder e biopolítica. Saúde Debate, 2016; 40(108): 178-89.

4. CABRAL JR, et al. Oficinas de educação em saúde com idosos: uma estratégia de promoção da qualidade de vida. Revista Enfermagem Digital Cuidado e Promoção da Saúde, 2015; 1(2): 71-75.

5. CAMATTA MW, et al. Ações de saúde mental na Estratégia Saúde da Família: Expectativas de familiares. Escola Anna Nery, 2016; 20(2): 281-88.

6. CARLOTTO MS, et al. Transtornos mentais comuns e associação com variáveis sociodemográficas e estressores ocupacionais: uma análise de gênero. Estudos e Pesquisas em Psicologia, 2015; 15(3): 1006-26.

7. CARVALHO MFAA, et al. Desarticulação da rede psicossocial comprometendo a integralidade do cuidado. Revista da Escola de Enfermagem da USP, 2017; 51: e03295.

8. CASTRO EAB, et al. Organização da atenção domiciliar com o Programa Melhor em Casa. Revista Gaúcha de Enfermagem, 2018; 39.

9. COPPOOLSE HL, et al. Impact of nutrition education on nutritional knowledge and intentions towards nutritional counselling in Dutch medical students: an intervention study. BMJ Open, 2020; 10(4): e034377.

10. DAMOUS I, ERLICH H. O ambulatório de saúde mental na rede de atenção psicossocial: reflexões sobre a clínica e a expansão das políticas de atenção primária. Physis Revista de Saúde Coletiva, 2017; 27(4): 911-32.

11. FARIAS DN, et al. Interdisciplinary and interprofessionality in the family health strategy. Trabalho, Educação e Saúde, 2018; 16(1): 141-62.

12. GIROLAMO G, et al. Is psychiatric residential facility discharge possible and predictable? A multivariate analytical approach applied to a prospective study in Italy. Soc Psychiatry Psychiatr Epidemiol, 2014; 49(1): 157-67.

13. GONZALES AM. Planos de Ações na Promoção e Prevenção de Transtornos em Saúde Mental na Atenção Primária à Saúde, BH. Monografia (Especialização em Atenção Básica em Saúde da Família). Universidade Federal de Minas Gerais. Contagem, 2015; 29 p.

14. HERMIDA PMV, et al. Active methodology in nursing education: innovation of the practice in the primary health care. Revista de Enfermagem da UFSM, 2015; 5(4): 683-91.

15. JORGE MSB, et al. The use of Active Methodologies in the process of Permanent Professional Qualification of workers to Operate in the Network of Integral Attention to Users of Crack and Other Drugs. Atas do Congresso Ibero-Americano de Investigação Qualitativa em Saúde, 2017; 2: 1204-13. 
16. KANTORSKI LP, et al. Alta dos usuários no centro de atenção psicossocial. Journal Nursing and Health, 2019; 9(3): e199305.

17. MCMINN BG, et al. Readiness for discharge from subacute mental health units for older people: using available measures to support clinical decision making. International Journal of Ment Health Nursing, 2017; 26(2): $151-9$.

18. MENDES VC, et al. Percepção sobre a alta hospitalar de pessoas internadas em unidade de Atenção Psicossocial. Revista Brasileira em Promoção da Saúde, 2019; 32: 93-94.

19. MESQUITA LLS, et al. Avaliação da qualidade dos serviços de saúde na atenção básica: correlação com o nível sociodemográfico e profissional das enfermeiras. International Journal of Development Research, 2020; 10(09): 40308-14.

20. MITRE SM, et al. Flexibilidade, acesso e diversidade na formação universitária em saúde: o aprender fazendo no desenvolvimento de competências profissionais. Modelos flexibles de la organización de la formación. Revista del Congrés Internacional de Docència Universitària i Innovació (CIDUI), 2014; 2: 1-15.

21. MORERA JAC, et al. Políticas e estratégias de redução de danos para usuários de drogas. Revista Baiana de Enfermagem, 2015; 29(1): 76-85.

22. PEIXOTO MM, et al. O encontro com a perspectiva do outro: empatia na relação entre psiquiatras e pessoas com diagnóstico de esquizofrenia. Ciência e Saúde Coletiva, 2016; 21(3): 881-90.

23. PINHEIRO LS, et al. Melhorias para a qualidade de vida e trabalho na visão dos agentes comunitários de saúde. Revista Brasileira de Medicina do Trabalho, 2019; 17(2): 180-7.

24. PIRES MRGM, et al. Fatores associados à atenção domiciliária: subsídios à gestão do cuidado no âmbito do SUS. Revista da Escola de Enfermagem da USP, 2013;7(3):648-56.

25. POLIT DF, BECK CT. Fundamentos de Pesquisa em Enfermagem: Avaliação de evidências para a prática da enfermagem. 7ed. Porto Alegre: Artmed; 2011.

26. POOLE R, et al. Delayed discharges in an urban in-patient mental health service in England. Psychiatric Bulletin, 2013; 34(4): 256-64.

27. QUEIROZ AHA, et al. Percepção de familiares e profissionais de saúde sobre os cuidados no final da vida no âmbito da atenção primária à saúde. Ciência e Saúde Coletiva, 2013; 18(9): 2615-23.

28. SAVASSI LCM. Os atuais desafios da atenção domiciliar na atenção primária à saúde: uma análise na perspectiva do Sistema Único de Saúde. Revista Brasileira de Medicina de Família e Comunidade, 2016; 1(38): 1-12.

29. SILVA BM, et al. O conhecimento dos agentes comunitários de saúde acerca da assistência em saúde mental no município de Macapá, Amapá. Revista Arquivos Científicos (IMMES), 2020; 3(2): 78-91.

30. SILVA-JUNIOR R, et al. O papel do agente comunitário de saúde no atendimento ao portador de transtorno mental. Revista Eletrônica Acervo Saúde, 2016; 8(1): 841-45.

31. SILVA LL, et al. The professionals' perspective on the patient discharge process of the Caps-AD: criteria and difficulties. Saúde Debate, 2019; 43(122): 819-35.

32. SILVA PF, et al. Conhecimento da equipe de enfermagem e agentes comunitários sobre o comportamento suicida. Revista de Enfermagem da UFPE, 2018; 12(1): 112-17.

33. SOUSA PF, et al. Evidências de validade fatorial e precisão da escala de atitudes em saúde mental. Estudos de Psicologia I, 2017; 34(1): 97-106.

34. SOUZA AC. Estratégias de inclusão da Saúde Mental na atenção básica: um movimento das marés. São Paulo: Hucitec, 2015; 26-27.

35. TRAJANO MP, et al. O cuidado em saúde mental: caminhos possíveis na rede de atenção psicossocial. Cadernos Brasileiros de Saúde Mental, 2018; 10(25): 20-37.

36. TRAPÉ TL, CAMPOS RO. Modelo de atenção à saúde mental do Brasil: análise do financiamento, governança e mecanismos de avaliação. Revista de Saúde Pública, 2017; 51(19).

37. VASCONCELOS MGF, et al. Projeto terapêutico em Saúde Mental: práticas e processos nas dimensões constituintes da atenção psicossocial. Interface: comunicação, saúde e educação, 2016; 20(57): 313-23.

38. XAVIER LN, et al. Analyzing active methods in the training of health professionals: an integrative review. International Journal of Development Research, 2014; 11(1): 43310-17.

39. ZANARDO GL, et al. Dispositivos e conexões da rede de atenção psicossocial (RAPS) de Porto Alegre - RS. Estudos Interdisciplinares em Psicologia. 2018; 9(3): 80-101. 